\title{
NONLINEAR EVOLUTION OF YUKAWA COUPLINGS IN THE DOUBLE HIGGS AND SUPERSYMMETRIC EXTENSIONS OF THE STANDARD MODEL
}

\author{
B. GRZADKOWSKI ' ${ }^{1}$ M. LINDNER ${ }^{2}$ and S. THEISEN \\ Max-Planck-Institut für Physik und Astrophysik - Werner-Heisenberg-Institut für Physik, \\ P.O. Box 401212, D-8000 Munich, Fed. Rep. Germany
}

Received 28 July 1987

\begin{abstract}
The nonlinear evolution equations for Yukawa coupling matrices are discussed in the context of the standard, double Higgs and supersymmetric model of electroweak interactions. In each case, even in the presence of a very heavy top quark the approximate analytical solutions for the nonlinear evolution of masses and mixing angles are found. Comparison of the analytical approach with numerical solutions confirm the excellent validity of the approximation. Some possible further applications of the developed method are suggested.
\end{abstract}

The renormalization group ( $R G$ ) equations provide an elegant and extremely useful tool for the study of grand unified theories (GUT's). Also, the pattern of masses and coupling parameters in the standard model might be explainable through the renormalization group flow of these parameters. Studying the experimental values of the gauge couplings with these ideas in mind typically leads to high unification scales $M_{\mathrm{X}}$ of the order of $10^{15} \mathrm{GeV}$. Predictions like $m_{\mathrm{b}} / m_{\tau} \approx 3$ are consequences of the linearized $\mathrm{RG}$ equations for Yukawa couplings [1]. As long as only light quarks $(<50 \mathrm{GeV})$ are considered linearization is a good approximation. But meanwhile heavy quarks seem to be realistic and therefore the nonlinearities become important. Up to now the full problem for an arbitrary number of generations is unsolved. There exist numerical studies for different quantities [2] and analytical solutions for some special problems [3].

In a recent paper [4] we have given analytical solutions for masses and mixing angles for the standard model (SM) with three generations. Here we extend our considerations to the case of the double

1 On leave of absence from Institute of Theoretical Physics, Warsaw University, PL-00681 Warsaw, Poland.

2 Address after September 1, 1987: Fermi National Accelerator Laboratory, P.O. Box 500, Batavia, IL 60510, USA.
Higgs (DHM) [5] and supersymmetric model (SUSYM) [6] of electroweak interactions. As previously we construct approximate analytical solutions for masses and mixing angles.

Since the method adopted here depends only on some coefficients which define the beta functions of the RG equations one can in fact generalize this approach to any theory with an analogous form of the beta functions. The solutions allow a discussion of the evolution of low energy parameters to arbitrary high scales varying not only unknown parameters but also the model. The possible structure of solutions is indeed much easier than expected from recent numerical simulations [7]. This is because the analytical solutions depend systematically only on special combinations of input parameters, a fact not easy to extract from numerical solutions.

The one-loop RG equations for Yukawa couplings are generally written as [8]

$$
\begin{aligned}
- & 16 \pi^{2} \mathrm{~d} M_{\mathrm{Y}}(t) / \mathrm{d} t \\
= & {\left[G_{\mathrm{Y}}(t)-T_{\mathrm{Y}}(t)-S_{\mathrm{Y}}(t)\right] M_{\mathrm{Y}}(t), }
\end{aligned}
$$

where $\mathrm{Y}=\mathrm{U}, \mathrm{D}, \mathrm{N}, \mathrm{E}$ stands for the corresponding quarks and leptons, respectively.

$M_{\mathrm{Y}}$ contains mass-matrices normalized in terms of vacuum expectation values $v_{i}$. In the standard 
model $v \simeq 175 \mathrm{GeV}$ defines the strength of the dimensionless elements in $M_{\mathrm{Y}}$. The other two models considered here have two VEV's related by $v_{1}^{2}+v_{2}^{2}=v^{2} \cdot v_{1}^{2}=r v^{2}$ and $v_{2}^{2}=(1-r) v^{2}$ with a free parameter $r$. In the following, we fix $r$ at the phenomenologically possible value $\frac{1}{2}$. ( $r$ close to 1 or 0 is excluded since then strong Higgs processes would show up in low-energy experiments.)

$G_{Y}$ contains the effective gauge coupling terms. As the one-loop $\beta$-function for the gauge couplings are decoupled they can be solved independently. In general we have

$G_{Y}=\sum_{i=1}^{3} K_{Y}^{i} g_{i}^{2}(t)$,

with the solution for $g_{i}^{2}(t)$ :

$g_{i}^{2}(t)=g_{i}^{2}(0) /\left[1-g_{i}^{2}(0) c_{i} t\right]$.

For the three models considered here the coefficients $K_{Y}^{i}$ and $c_{i}$ are given in tables 1 and 2 ( $N$ is the number of generations).

In eq. (1) $T_{Y}$ denotes trace terms:

$$
\begin{aligned}
& T_{\mathrm{U}}=\operatorname{Trace}\left(3 M_{\mathrm{U}} M_{\mathrm{U}}^{+}+3 a M_{\mathrm{D}} M_{\mathrm{D}}^{+}\right. \\
& \left.\quad+M_{\mathrm{N}} M_{\mathrm{N}}^{+}+a M_{\mathrm{E}} M_{\mathrm{E}}^{+}\right), \\
& T_{\mathrm{D}}=\operatorname{Trace}\left(3 a M_{\mathrm{U}} M_{\mathrm{U}}^{+}+3 M_{\mathrm{D}} M_{\mathrm{D}}^{+}\right. \\
& \left.\quad+a M_{\mathrm{N}} M_{\mathrm{N}}^{+}+M_{\mathrm{E}} M_{\mathrm{E}}^{+}\right), \\
& T_{\mathrm{N}}=T_{\mathrm{U}}, \quad T_{\mathrm{E}}=T_{\mathrm{D}},
\end{aligned}
$$

$a$ are numbers given in table 3 . The last term in eq. (1) is $S_{\mathrm{Y}}$.

Table 1

$K_{Y}^{i}$ for SM, DHM and for SUSYM.

\begin{tabular}{lllll}
\hline Model & Y & \multicolumn{1}{l}{$i$} & \\
\cline { 3 - 4 } & & 1 & 2 & 3 \\
\hline SM, DHM & U & $\frac{17}{12}$ & $\frac{9}{4}$ & 8 \\
& D & $\frac{5}{12}$ & $\frac{9}{4}$ & 8 \\
& N & $\frac{3}{4}$ & $\frac{9}{4}$ & 0 \\
& E & $\frac{15}{4}$ & $\frac{9}{4}$ & 0 \\
SUSYM & & & & \\
& U & $\frac{13}{9}$ & 3 & $\frac{16}{3}$ \\
& D & $\frac{7}{9}$ & 3 & $\frac{16}{3}$ \\
& N & 1 & 3 & 0 \\
& E & 3 & 3 & 0 \\
\hline
\end{tabular}

Table 2

$c_{i}$ coefficients in units of $1 / 8 \pi^{2}$.

\begin{tabular}{llll}
\hline Model & $i$ & & \\
\cline { 2 - 4 } & 1 & 2 & 3 \\
\hline SM & $\frac{20}{9} N+\frac{1}{6}$ & $\frac{4}{3} N-\frac{43}{6}$ & $\frac{4}{3} N-11$ \\
DHM & $\frac{20}{9} N+\frac{1}{3}$ & $\frac{4}{3} N-7$ & $\frac{4}{3} N-11$ \\
SUSYM & $\frac{10}{3} N+1$ & $2 N-5$ & $2 N-9$ \\
\hline
\end{tabular}

$S_{\mathrm{U}}=\frac{3}{2}\left(b M_{\mathrm{U}} M_{\mathrm{U}}^{+}+c M_{\mathrm{D}} M_{\mathrm{D}}^{+}\right)$,

$S_{\mathrm{D}}=\frac{3}{2}\left(b M_{\mathrm{D}} M_{\mathrm{D}}^{+}+c M_{\mathrm{U}} M_{\mathrm{U}}^{+}\right)$,

$S_{\mathrm{E}}=\frac{3}{2}\left(b M_{\mathrm{E}} M_{\mathrm{E}}^{+}+c M_{\mathrm{N}} M_{\mathrm{N}}^{+}\right)$,

$S_{\mathrm{N}}=\frac{3}{2}\left(b M_{\mathrm{N}} M_{\mathrm{N}}^{+}+c M_{\mathrm{E}} M_{\mathrm{E}}^{+}\right)$.

In table 3 the numbers $a, b, c$ are given for the three models considered.

At this point we specialize to $N=3$ and make the approximation where all contributions to $T_{\mathrm{Y}}$ and $S_{\mathrm{Y}}$ are due to a heavy top quark. With this approximation we get

$$
\begin{aligned}
& T_{\mathrm{U}}=T_{\mathrm{N}} \simeq 3 g_{\mathrm{t}}^{2}, \quad T_{\mathrm{D}}=T_{\mathrm{E}} \simeq 3 a g_{\mathrm{t}}^{2}, \\
& S_{\mathrm{U}} \simeq \frac{3}{2} b g_{\mathrm{t}}^{2} E_{3}, \quad S_{\mathrm{D}} \simeq \frac{3}{2} c g_{\mathrm{t}}^{2} E_{3}, \quad S_{\mathrm{E}}=S_{\mathrm{N}} \simeq 0, \\
& E_{13}=\left(\begin{array}{lll}
0 & 0 & 0 \\
0 & 0 & 0 \\
0 & 0 & 1
\end{array}\right) .
\end{aligned}
$$

Before we write down the solutions of eq. (1) with the above approximation we define

$$
\begin{aligned}
& \alpha_{\mathrm{Y}}\left(t_{1}, t_{2}\right)=\exp \left(-\frac{1}{16 \pi^{2}} \int_{t_{1}}^{t_{2}} G_{\mathrm{Y}}(\tau) \mathrm{d} \tau\right), \\
& \beta\left(t_{1}, t_{2}\right)=\exp \left(\frac{3}{16 \pi^{2}} \int_{t_{1}}^{t_{2}} g_{\mathrm{t}}^{2}(\tau) \mathrm{d} \tau\right) .
\end{aligned}
$$

Table 3

$a, b$ and $c$ of eqs. (7)-(10) for the models considered.

\begin{tabular}{lllr}
\hline Model & $a$ & $b$ & $c$ \\
\hline SM & 1 & 1 & -1 \\
DHM & 0 & 1 & $\frac{1}{3}$ \\
SUSYM & 0 & 2 & $\frac{2}{3}$ \\
\hline
\end{tabular}


Note that $\alpha_{Y}$ and $\beta$ are not always identical to $\alpha_{Y}$ and $\beta$ in ref. [4] as the coefficients $K_{Y}^{i}, c_{i}$ and $a, b$, $c$ depend on the model.

Applying the same method as in ref. [4] we solve eq. (1) for small mixing angles and arbitrary phase. The solutions are

$$
\begin{aligned}
& g_{\mathrm{b}}\left(t_{\mathrm{X}}\right)=\alpha_{\mathrm{D}} \beta^{a+c / 2} \sqrt{1+\Delta^{2} L_{\mathrm{b}}} g_{\mathrm{b}}\left(t_{\mathrm{Z}}\right), \\
& g_{\mathrm{s}, \mathrm{d}}\left(t_{\mathrm{X}}\right)=\alpha_{\mathrm{D}} \beta^{\alpha} \sqrt{1+\Delta^{2} L_{\mathrm{s}, \mathrm{d}}} g_{\mathrm{s}, \mathrm{d}}\left(t_{\mathrm{Z}}\right), \\
& g_{\mathrm{t}}\left(t_{\mathrm{X}}\right)=\alpha_{\mathrm{U}} \beta^{(b+2) / 2} g_{\mathrm{t}}\left(t_{\mathrm{Z}}\right), \\
& g_{\mathrm{u}, \mathrm{c}}\left(t_{\mathrm{X}}\right)=\alpha_{\mathrm{U}} \beta g_{\mathrm{u}, \mathrm{c}}\left(t_{\mathrm{Z}}\right), \\
& g_{\mathrm{e}, \mu, \tau}\left(t_{\mathrm{X}}\right)=\alpha_{\mathrm{E}} \beta^{a} g_{\mathrm{e}, \mu, \tau}\left(t_{\mathrm{Z}}\right), \\
& g_{v_{c}, v_{\mu}, v_{\tau}}\left(t_{\mathrm{X}}\right)=\alpha_{\mathrm{N}} \beta g_{v_{c}, v_{\mu}, v_{\tau}}\left(t_{\mathrm{Z}}\right) .
\end{aligned}
$$

Here the $L$ are model independent and therefore identical to the quantities defined in ref. [4]:

$L_{\mathrm{b}}=\left(1-2 \Delta^{-1}\right)|f|^{2}$,

$L_{\mathrm{s}}=-L_{\mathrm{b}} /\left(1+\Delta^{2} L_{\mathrm{b}}\right)$,

$L_{\mathrm{d}}=0$,

where $\Delta=1-\beta^{-c / 2} \cdot|f|^{2}=\left|\theta_{2}+\alpha_{3} \mathrm{e}^{\mathrm{i} \delta}\right|^{2}$ is the only relevant mixing angle dependence.

To leading order in small quantities the evolved mixing angles are

$\theta_{1}\left(t_{\mathrm{X}}\right)=\theta_{1}\left(t_{\mathrm{Z}}\right)+\mathrm{O}(3)$,

$\theta_{2}\left(t_{\mathrm{X}}\right)=\theta_{2}\left(t_{\mathrm{Z}}\right) \beta^{-c / 2}+\mathrm{O}(2)$,

$\theta_{3}\left(t_{\mathrm{X}}\right)=\theta_{3}\left(t_{\mathrm{Z}}\right) \beta^{-c / 2}+\mathrm{O}(2)$

$\delta\left(t_{\mathrm{X}}\right)=\delta\left(t_{\mathrm{Z}}\right)+\mathrm{O}(2)$,

where the orders of the next corrections are indicated by $O(\ldots)$. To summarize the results we note that the model specifications enter in four ways:

(a) through a modified gauge evolution (coefficients $c_{i}$ in eq. (3));

(b) through a modified contribution of the gauge couplings to the evolution of Yukawa couplings (coefficients $K_{i}^{Y}$ in eq. (2) and in $\alpha_{Y}$ of eq. (14));

(c) through a modified $\beta$, depending on all coefficients;

(d) through a dependence of the solutions eqs. (15) on powers of $\beta$ given by the coefficients $a, b$ and $c$.

For a quantitative discussion we evaluate $\beta$ now numerically. By solving eq. (14) $\alpha_{Y}$ is given analytically through $\int_{t_{1}}^{t_{2}} g_{i}^{2}(\tau) \mathrm{d} \tau=-\frac{1}{c_{i}} \ln \left(\frac{1-g_{i}^{2}(0) c_{i} t_{2}}{1-g_{i}^{2}(0) c_{i} t_{1}}\right)$.

Note that $0<\alpha_{Y} \leqslant 1$ and $\beta \geqslant 1$.

It turns out that our approximate analytical solutions are so precise that we can present them, within resolution, together with the numerical solutions, by one curve only. Therefore the whole discussion of results will be performed in the language of the analytical solutions. Since in the presence of the heavy top quark the Yukawa couplings (as being proportional to mass) play a crucial role, some remarks concerning Higgs corrections to the Yukawa couplings of the neutral scalars are in order here. As we work in the mass-independent renormalization scheme (or in the ultraviolet limit) all masses can be neglected. Therefore in each considered model one observes cancellation of vertex corrections induced by real and imaginary parts of the neutral components of the Higgs doublets. This is a simple consequence of the additional factor ' $i$ ' which appears in couplings of imaginary parts to fermions. As can be easily checked the same cancellation does not occur in corrections to external legs since $g_{\mathrm{Y}} g_{\mathrm{Y}}^{+}$always appears and contributions from real and imaginary parts enter with the same sign. The next remark concerns the charged Higgs contributions to vertex corrections. Since in the SUSYM and DHM each quark doublet couples to only one of the Higgs doublets (this is automatic in the SUSYM and arranged such as to avoid flavour changing neutral currents (FCNC) in the DHM) it is easy to see that in these models there are no virtual charged scalars in vertex corrections - the exchange of a charged Higgs induces in a loop a fermion of different charge to which an external neutral scalar does not couple.

As we learn from eqs. (15)-(17) the most transparent difference between the SM and SUSYM or DHM consists of the fact that in the former the parameter $c$ is negative whereas in SUSYM and DHM $c$ is positive. This fact explains why the heavy top effects are smaller for $g_{\mathrm{b}}$ than for $g_{\mathrm{d}}$ and $g_{\mathrm{s}}$ in the SM (additional negative power of $\beta^{+c / 2}-$ see eq. (15)). Since we have $c>0$ for SUSYM and DHM we observe in these cases an opposite behaviour (fig. 1a). As is seen from our solutions, the sign of $c$ is also responsible for a different evolution of mixing angles 

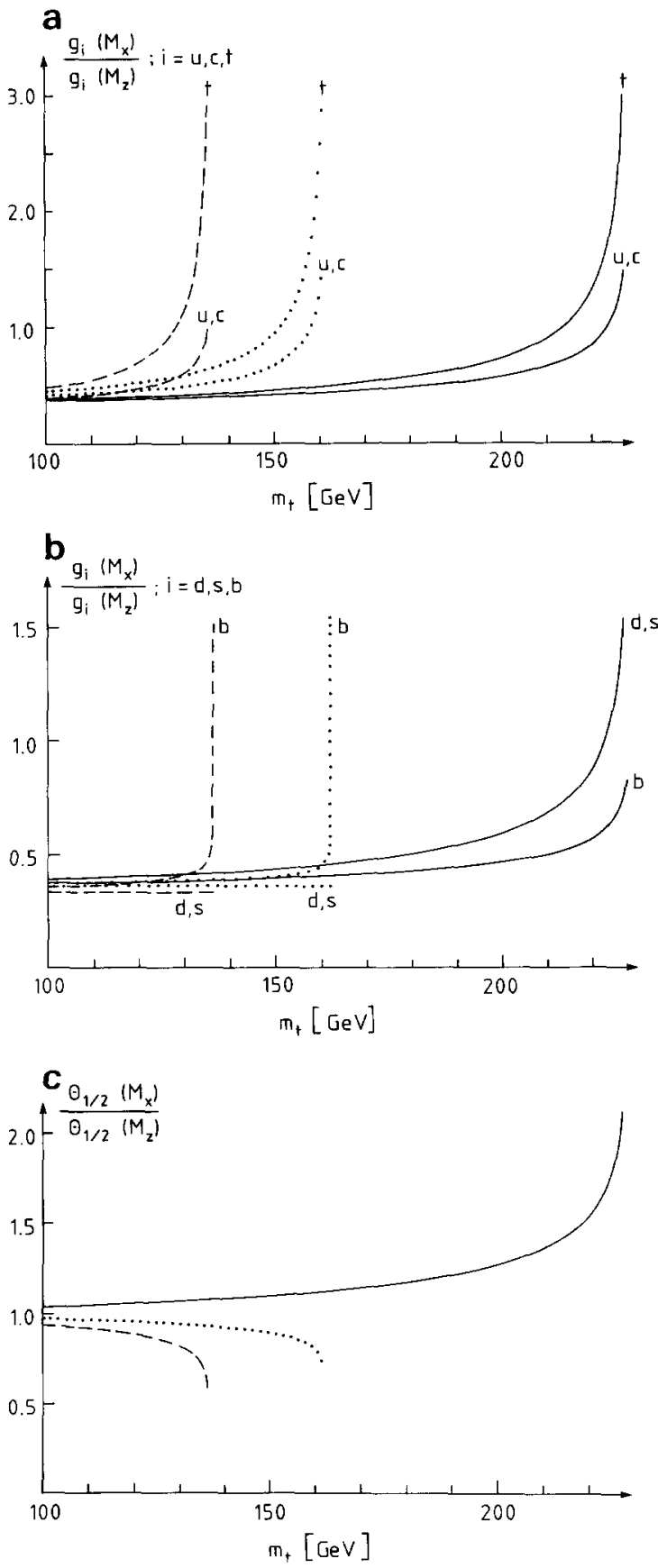

Fig. 1. The result of the evolution for the (a) up-type (b) downtype and (c) the Kobayashi-Maskawa mixing angles $\theta_{i}$ as a function of $m_{1}$. The end points of each curve correspond to the pole in the evolution of $g_{1}$. The solid, dotted and dashed lines correspond to the standard, double Higgs and supersymmetric model of the weak interactions, respectively. (fig. 1c) - in the SM a heavy top increases $\theta_{2 / 3}\left(M_{\mathrm{X}}\right)$ whereas in the other models it leads to a decrease. Let us try to find the source of these differences. Looking at individual diagrams one easily notices that fermion loops (contributing to $T_{\mathrm{Y}}$ ) and corrections to the external quark legs have the same sign and cause increasing heavy top effects. Only vertex corrections with a virtual charged scalar enter with an opposite (negative) sign. However as we recall these diagrams appear only in the SM. It turns out that in this case they are dominant and result in $c<0$. As these diagrams are absent in the other cases we have $c>0$ in the SUSYM and DHM.

The positive sign of $c$ is a direct consequence of natural suppression of FCNC in models where such currents could in principle appear (DHM). The fact that for SUSYM $c$ (and also $b$ ) is twice the value in the DH model reflects the new contributions coming from superpartners of ordinary particles appearing in loops (external quark-leg corrections).

In fig. $1 \mathrm{~b}$ we present the results for the evolution of the up-type Yukawa couplings. As we see heavy top effects are bigger for $g_{\mathrm{t}}$ than for $g_{\mathrm{u}}$ and $g_{\mathrm{c}}$. In terms of our solutions it is the result of a positive $b$ (for all considered models). Such a behaviour is clear as only neutral scalars can produce heavy top effects in the evolution of $g_{1}$ and as we already know these contributions enter always with the same positive sign. It is remarkable that the behaviour of $g_{\mathrm{u}}$ and $g_{\mathrm{c}}$ are exactly the same (as was predicted by our solutions eq. (15)) - the small intergeneration mixing is responsible for this effect (the same is also true for the evolution of the down-type Yukawa couplings). Comparing figs. $1 \mathrm{a}$ and $1 \mathrm{~b}$ it is seen that the heavy top effects are smaller in the case of $g_{\mathrm{b}}$ than $g_{\mathrm{t}}$. It is related to the fact that in the case of $g_{\mathrm{b}}$ we observe a kind of destructive interference between diagrams contributing to the trace $T_{\mathrm{Y}}$ and those which give $S_{\mathrm{Y}}$ in the beta function of eq. (1). Also in this case it is related to the presence of the vertex corrections from charged scalars for $g_{\mathrm{b}}$ which enter with a negative sign. As we explained, such diagrams do not appear for $g_{\mathrm{t}}$.

In fig. 1c we also see how precisely our analytical solutions work; all predictions (eqs. (17) for $\theta_{i}\left(M_{\mathrm{X}} / \theta_{i}\left(M_{\mathrm{Z}}\right)\right.$ are satisfied - no evolution of $\theta_{1}$ and exactly the same behaviour for $\theta_{2}$ and $\theta_{3}$. The following remark is in order here. As we know from ex- 


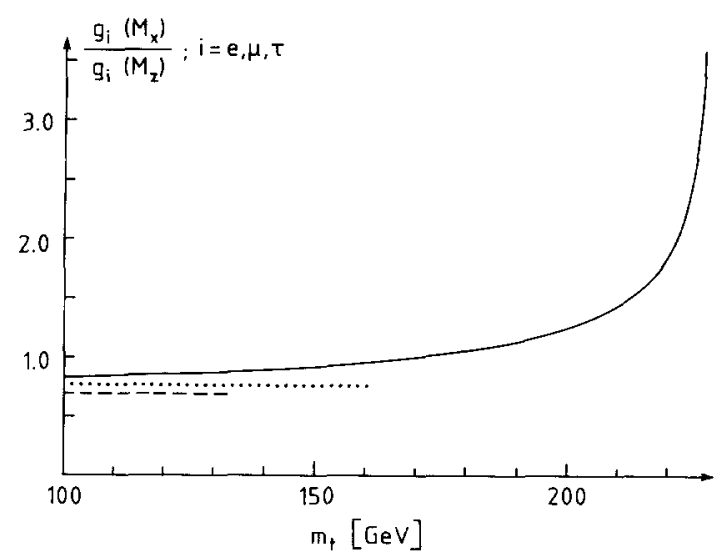

Fig. 2. The running of the lepton Yukawa couplings as a function of $m_{\mathrm{t}}$. The end points and the labelling of the curves is as in fig. 1.

periments $\theta_{2}$ and $\theta_{3}$ are small angles. It is easy to understand the peculiar conservation of $\theta_{1}$ just in the limit of $\theta_{2 / 3} \rightarrow 0$ namely from the general solution for the down type sector (eqs. (11), (12)) we see that without any additional approximation we get an exact conservation of the $\mathrm{K}-\mathrm{M}$ matrix ([ $E_{3}$, $\left.\left.U_{\mathrm{K}-\mathrm{M}}\left(t_{\mathrm{Z}}\right)\right]=0\right)$, which means that $\theta_{1}\left(M_{\mathrm{X}}\right)=\theta_{1}\left(M_{\mathrm{Z}}\right)$ and $\delta\left(M_{\mathrm{X}}\right)=\delta\left(M_{\mathrm{Z}}\right)$. The last qualitative prediction is in fact consistent with our systematic expansion (eq. (17)), as should be since we have treated the mixing angles as small quantities.

For completeness, in fig. 2 we present also the result for the evolution of leptons. As indicated by our equations the behaviour of $e, \mu$ and $\tau$ is exactly the same since heavy top effects energy enter only through the trace $T$ (eqs. (6), (9), (10)) producing an universal factor for all families. Let us remark that due to the mentioned cancellation the running of leptons in even bigger than for $g_{\mathrm{b}}$ coupling. Additionally the QCD suppression is not acting on leptons.

To summarize, we have found very precise analytical situations of the RG equations for Yukawa coupling matrices. Our solutions, having a simple form allow easily to answer questions about nonlinear evolution of masses and mixing angles between arbitrary scales in the standard, double Higgs and supersymmetric model of electroweak interactions. The method adopted here is general enough to be applied to other models with a similar structure of the beta function. A possible application of our approach consists mostly in a simplified construction of theories of grand unification. Our formulas can be used to put strong constraints on a particular GUT. The restrictions are parametrized by $m_{\mathrm{t}}$ and also by some not precisely measured low-energy quantities like light quark masses or some mixing angles. The remaining degrees of freedom would either allow to match the conditions or rule out the model. An opposite application is certainly also possible. Assuming some embedding scenario one can obtain some information about the parameters of the evolution $\left(m_{\mathrm{t}}, \ldots\right)$ fitting high-energy predictions to low-energy experimental data.

\section{References}

[1] A.J. Buras, J. Ellis, M.K. Gaillard and D.V. Nanopoulos, Nucl. Phys. B 135 (1978) 66.

[2] J. Bagger, S. Dimopoulos and E. Masso, Nucl. Phys. B 253 (1985) 397

K. Sasaki, Z. Phys. C 32 (1986) 149.

[3] S. Dimopoulos and S. Theodorakis, Phys. Lett. B 154 (1985) 153.

[4] B. Grzadkowski and M. Lindner, Phys. Lett. B 193 (1987) 71

[5] R.A. Flores and M. Sher, Ann. Phys. (NY) 148 (1983) 95, and references therein.

[6] M. Dine, W. Fischler and M. Srednicki, Nucl. Phys. B 189 (1981) 575

S. Dimopoulos and S. Raby, Nucl. Phys. B 192 (1981) 353;

S. Dimopoulos and H. Georgi, Nucl. Phys. B 193 (1981) 150; N. Sakai, Z. Phys. C 11 (1981) 153.

[7] K.S. Babu, Z. Phys. C 35 (1987) 69.

[8] T.P. Cheng, E. Eichten and L.-F. Li, Phys. Rev. D 9 (1974) 225. 